\title{
Sistema de monitoreo remoto de acuicultura en estanques para la crianza de camarones
}

\author{
Remote monitoring system of aquaculture in tanks for shrimp breeding
}

\author{
Susana Flores Mollo ${ }^{1} \quad$ Diego Aracena Pizarro ${ }^{1 *}$
}

Recibido 27 de junio de 2018, aceptado 06 de agosto de 2018

Received: June 27, 2018 Accepted: August 06, 2018

\begin{abstract}
RESUMEN
La calidad del agua de un estanque en el campo de la acuicultura tiene un papel muy importante para el éxito del cultivo o crianza de camarones. Los parámetros del agua son variables que pueden afectar la salud del animal o afectar en un crecimiento tardío y una reproducción lenta. Los parámetros del agua se miden comúnmente de manera manual con la ayuda de instrumentos de medición para conocer su estado. Los datos de las mediciones permiten notar cambios y tomar decisiones ante emergencias. Se desarrolla un sistema de monitoreo remoto, con el objetivo de automatizar el proceso de toma de datos y evitar accidentes con las especies en observación, éste incluye nodos con sensores para medir parámetros del agua, sensores ambientales, módulo de comunicación inalámbrica XBee y microcontrolador Arduino. El trabajo en conjunto permite la toma de lecturas programadas o en tiempo real. La información de cada lectura tomada de los parámetros puede ser consultada desde un dispositivo móvil o un computador con acceso a internet en cualquier instante. Finalmente se realizan pruebas del sistema, para comprobar su funcionamiento en el ambiente requerido. Se instalan nodos en cada estanque del "proyecto piloto de criaderos de camarones".
\end{abstract}

Palabras clave: Monitoreo, parámetros del agua, sensores, microcontrolador Arduino, XBee.

\begin{abstract}
The water quality of a pond in the field of aquaculture has a very important role in the success of growing or raising shrimp. Water parameters are variables that can affect the health of crustaceous or affect late growth and slow reproduction. Water parameters are commonly measured manually with the help of measuring instruments to determine their status. Measurement of data allows to note changes and make decisions in emergencies. A remote monitoring system is developed, with the objective of automating the process of data capture and avoiding accidents with the species under observation, this includes nodes with sensors to measure water parameters, environmental sensors, XBee wireless communication module and Arduino microcontroller. Working together allows the taking of scheduled readings or in real time. The information of each reading taken from the parameters can be consulted from a mobile device or a computer with internet access at any time. Finally, the system is tested to verify its operation in the required environment. Nodes are installed in each pond of the "pilot project of shrimp farms".
\end{abstract}

Keywords: Monitoring, water parameters, sensors, Arduino microcontrollers, XBee.

1 Escuela Universitaria de Ingeniería Industrial, Informática y Sistemas, Área de Ingeniería de Computación e Informática. Universidad de Tarapacá. Arica, Chile.E-mail: susanafloresmollo22@gmail.com,daracena@academicos.uta.cl

* Autor de correspondencia. 


\section{INTRODUCCIÓN}

La acuicultura está definida por ley en Chile, como la "Actividad que tiene por objeto la producción de recursos hidrobiológicos organizada por el hombre". Esta actividad ha tomado fuerza en las últimas décadas, principalmente con el cultivo del salmón en el sur, llegando a ser Chile unos de los principales exportadores a nivel mundial. La acuicultura nacional factura más de USD $\$ 4.500$ millones, emplea más de 45.000 personas y ha sido uno de los de mayor crecimiento en los últimos 30 años, como se puede ver en la Figura 1.

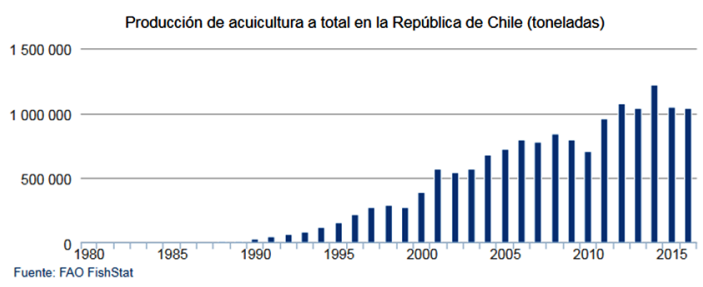

Figura 1. Producción de la Acuicultura reportada en Chile. Fuente: [1].

Sin embargo, el sector de producción de salmónidos vive actualmente una crisis sanitaria que ha obligado a disminuir la producción y todo lo que esto conlleva, ya que representa el 93\% de las ventas, dejando de manifiesto la necesidad de explorar en otras áreas y planificar estrategias de desarrollo paralelas. En ese contexto, se ha establecido que para mantener altas tasas de crecimiento de la acuicultura y consolidar al país como líder mundial del sector es importante el aporte de la diversificación de nuevas especies para el cultivo, en donde se menciona el Camarón de río del norte de Chile "Cryphiops caementarius" [2].

Para que una especie pueda ser reproducida es necesario encontrar condiciones ideales del agua, por esta razón, se pretende abordar este problema, desarrollando un sistema de monitoreo web del agua de los estanques, con el principal objetivo que pueda ser una herramienta de apoyo a la acuicultura de pequeña escala en la región de Arica y Parinacota.

En la zona norte del país la acuicultura es realizada en menor cantidad, no obstantes existe un número considerable de proyectos de cultivos que necesitan de alguna forma mejorar sus procesos, ya que, así aumentarían su productividad. Para el proyecto piloto de criadero de camarones el principal beneficio sería que, al poder mantener el control de una mayor cantidad de estanques (hatchery), aumentaría la cantidad de camarones reproducidos, demostrando que en la zona norte del país también es posible el desarrollo de la acuicultura aplicando tecnología y conocimiento en distintas áreas en conjunto. También, que la realización de un cultivo masivo de una especie autóctona de la ciudad para la venta es posible.

En la actualidad se han implementado sistemas de monitoreo en la acuicultura, como en el cultivo de microalgas que se lleva acabo al oeste de noruega, el objetivo del estudio fue investigar la aplicación de sensores de transmisión de luz, para el monitoreo y control de densidad de la producción de biomasa de microalgas en reactores tubulares de gran escala. Como resultado se encontraron patrones de crecimiento y variables de entorno que maximizan la producción de microalgas gracias al monitoreo constante [3].

K. Bhuiya, K. Anish, D. Parekh y K. Laxmi [4] presenta un sistema de monitoreo y control de temperatura y nivel agua de un estanque, sin intervención humana, de una manera simple y bajo costo, utilizando lógica programable PLC y red inalámbrica Zigbee. Siendo ésta una tecnología inalámbrica desarrollada como un estándar global abierto, para hacer frente a las necesidades únicas de las redes de área personal inalámbricas de baja potencia y de bajo costo (WPAN). Este estándar saca el máximo partido a la especificación de la capa física IEEE 802.15.4 y opera en bandas sin licencia en todo el mundo en las siguientes frecuencias: $2.400-2.484 \mathrm{GHz} 902-928 \mathrm{MHz}$ y 868,0 - 868,6 MHz. ZigBee puede conformar diferentes tipos de redes, tales como: en la red en estrella el coordinador ZigBee inicia la red y todos los otros nodos están asociados directamente con el coordinador ZigBee. En los otros dos tipos de redes hay un tercer tipo de nodos ZigBee, routers ZigBee o el coordinador ZigBee, a las que los dispositivos finales se pueden asociar. En las redes de árboles los routers ZigBee forman un árbol que tiene sus raíces en el coordinador ZigBee.

Un trabajo de aplicación de visión computacional en la acuicultura para el monitoreo del tamaño de peces 
$\mathrm{u}$ otros seres vivos, se presenta en Boaz Zion [5], en donde los sujetos inspeccionados son sensibles, fácilmente estresables y libre de moverse en un entorno en donde la visibilidad, iluminación y estabilidad no son generalmente controlables, donde los sensores deben funcionar en un ambiente húmedo. Una publicación reciente de Mathiassen, Misimi, Bondo; Veliyulin y Ostvik [6] estudiaron la posibilidad de diversas tecnologías de imagen, incluyendo la visión computacional, para la inspección de los peces y productos pesqueros. Se miraron cuatro grandes áreas de aplicación: la investigación, la comprensión y optimización de procesos, selección y clasificación automatizada, y el procesamiento automatizado de las operaciones pos-cosecha, a través de máquinas de visión. Mediante procesamiento morfológico de las imágenes se puede contar, estimar tamaño y masa, identificar especímenes para la evaluación de calidad y controlar el bienestar de las especies.

Este trabajo presenta un sistema que permite agilizar la captura de datos de los estanques, de manera programada y continua. Es así, como se obtiene una mayor cantidad de datos para el análisis del comportamiento de los parámetros del agua, eliminando el trabajo manual, con instrumentos de medición y registro en hojas de papel.

\section{EL CAMARÓN CRYPHIOPS CAEMENTARIUS}

\section{Morfología corporal}

Animal robusto, de abdomen tan largo y grueso como el cefalotórax, rostrum con cresta dorsal adornada por fila de 6 a 7 dientes gruesos, puede tener dientes a lo largo del borde ventral o carecer completamente de ellos, existiendo ejemplares en todos los estados intermedios relativos a estos extremos. Primer y segundo par de patas caminadoras con quela o tenaza terminal, el segundo par mucho más grande que el primero y una de las patas de mayor tamaño que la opuesta. El segundo par de patas del macho es distinto al de la hembra, la mayor anchura de los extremos del segundo segmento abdominal, en proporción a la longitud del abdomen y la relación cefalotoráxica, nos dan la evidencia de un dimorfismo sexual [7].

\section{Distribución geográfica}

Se distribuye en los ríos de la costa occidental del Perú y Chile. En Chile llega hasta Valparaíso como límite Sur. Desde la Región de Arica y Parinacota a la Región de Valparaíso. Registrado en ríos y quebradas desde Tejas Verdes (Río Maipo) hacia el norte. Se encuentra en los ríos Loa, Elqui, Huasco, La Ligua, Aconcagua. También se encuentra en el río Lluta y Camarones en Arica [7].

\section{Condiciones de mantención del cultivo de larvas}

Los principales antecedentes del cultivo de esta especie en la etapa larval como se puede ver en la Figura 2, se remontan a la década de los sesenta, donde se comenzó a nivel experimental los ensayos para la determinación de las condiciones óptimas de cultivo, con el fin de obtener la máxima supervivencia en condiciones controladas.

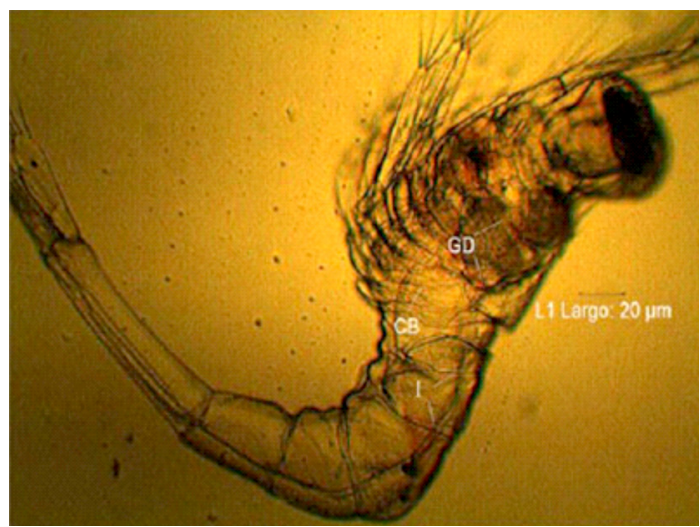

Figura 2. Vista lateral de una larva de Cryphiops caementarius. Fuente: [8].

Los trabajos realizados en Perú y Chile determinan para el camarón, las siguientes condiciones de operación y mantención del cultivo:

Temperatura del agua: La mayoría de los trabajos experimentales de cultivo se han realizado con temperaturas que oscilaron entre los $20^{\circ} \mathrm{C}$ y $25^{\circ} \mathrm{C}$.

Oxígeno disuelto: Los valores de análisis de oxígeno disuelto en el agua de cultivo o sistemas de airación y recambio de agua, sugieren trabajar siempre con aguas saturadas de oxígeno, considerándose como valores aceptables de 7 a $10 \mathrm{mg} / \mathrm{l}$.

Potencial de hidrógeno: Antecedentes de análisis de $\mathrm{pH}$ del medio de cultivo para larvas, los valores mínimos y máximos permisible son de 7 a 8.6, siendo el óptimo de 7.5 a 8.0. 
Amonio, nitritos y nitratos: Existen escasos registros claros de los máximos permisibles para el cultivo de larvas de C. caementarius. No obstante, en términos generales los límites máximos corresponden a: amonio $0.15 \mathrm{ppm}$; nitritos $0.25 \mathrm{ppm}$ y nitratos $4.0 \mathrm{ppm}$.

Salinidad: Usaron en una primera experiencia valores de salinidad correspondientes a: 0, 10, 20, 30 y $40 \%$, encontrando la mayor supervivencia al $30 \%$.

Estos son los parámetros del agua que se consideraron en los trabajos realizados de cultivo de larvas de camarón [7]. Estudios más recientes acotan el mínimo y máximo de la temperatura del agua para las larvas entre $24^{\circ} \mathrm{C}$ a $26^{\circ} \mathrm{C}$, donde se consiguió $7,4 \%$ de supervivencia del total [8]. El camarón puede vivir con concentraciones mínimas de oxígeno, de hasta 4 ppm, sin embargo, los valores óptimos para su cultivo están entre 7.0 y 8.4 ppm [9].

\section{ARQUITECTURA DEL SISTEMA}

La arquitectura del sistema es del tipo clienteservidor, en donde el cliente, es representado por un notebook y dispositivo móvil. Cualquier dato que se desee solicitar debe pasar por el servidor. El sistema consta de cuatro subsistemas que son: subsistema monitoreo y alertas, subsistema recolector de datos serial, subsistema nodo recolector de datos y subsistema de almacenamiento de datos (servidor). El subsistema "Nodo recolector de datos" corresponde a un nodo sensor, compuesto por un microcontrolador, sensores y el dispositivo XBee de comunicación inalámbrica. En la Figura 3 se puede observar cómo se conectan estos subsistemas.

En donde el sistema está compuesto por los siguientes subsistemas:

- Subsistema "Nodo recolector de datos": Es un nodo sensor que está conformado por un microcontrolador "Arduino", que se encarga de procesar la información obtenida de los sensores y de transmitir los datos a través de un transceptor. El sistema puede soportar más de un nodo recolector de datos, por esta razón los nodos son identificados por un ID. En el Arduino se ejecuta un programa en donde se

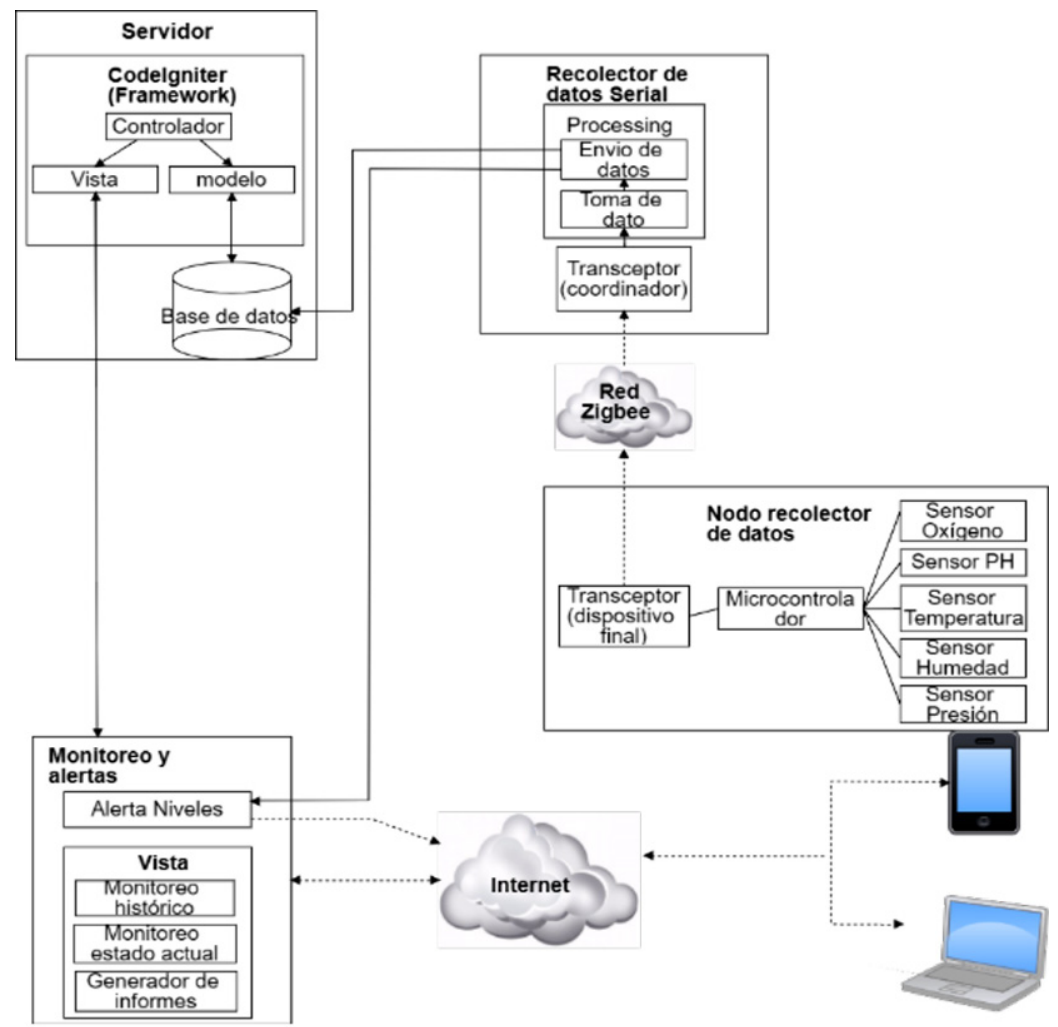

Figura 3. Arquitectura del sistema de monitoreo. Fuente propia. 
asignan el ID, también se inicializan los sensores y se ordena que capturen los datos sólo si el nodo ha recibido el mensaje de petición del subsistema correspondiente.

- Subsistema "recolector de datos serial": Este subsistema se encarga de solicitar los datos, al nodo recolector de datos a través de un programa realizado en el entorno de desarrollo Processing [10]. Éste programa facilita la escucha del puerto serial, por donde entran los datos enviados del nodo sensor, el cual se ejecuta en el computador que actúa de servidor. Por uno de los puertos USB se conecta un transceptor XBee, éste es el coordinador de la red inalámbrica, envía las peticiones que se ejecutan en Processing.

- El subsistema tiene dos modalidades de solicitud de datos que ejecuta Processing.

- Solicitud de datos automática: Se programa para que solicite datos al nodo sensor cada 15 minutos.

- Solicitud de datos manual: Se lee un archivo .txt, donde el subsistema de monitoreo y alertas escribe una petición de datos de algún estanque seleccionado en la interfaz de usuario de algún cliente, para saber el estado actual del estanque. Esto puede suceder en cualquier instante del día.

- Subsistema de "Almacenado de datos": El encargado de insertar los valores que son enviados desde el subsistema recolector de datos seriales, en las tablas de una base de datos MYSQL. Además, ejecuta las solicitudes de envió de datos masivos desde el subsistema de monitoreo y alerta, para generar gráfico o informes.

- Subsistema "Monitoreo y alerta": Para que el usuario pueda monitorear los datos de manera local o remota, este subsistema hace referencia a las vistas hechas en CodeIgniter, un framework PHP, para el desarrollo de la aplicación con interfaz gráfica usuario web. Muestra el estado actual de los estanques, historial de las variables medidas del agua y ambiente, configuración de niveles mínimos y máximos, selección de datos para generación de informes e historial de notificaciones de alerta de máximos y mínimos. También notifica al correo electrónico de los usuarios registrados en el sistema si las variables medidas han sobrepasado los niveles mínimos y máximos, establecidos en la configuración.

\section{Área de trabajo}

$\mathrm{La}$ arquitectura del sistema descrito anteriormente, se diseñó tomando en cuenta el área de trabajo que se presenta en las Figuras 4 y 5, correspondientes al laboratorio del "proyecto piloto de criaderos de camarones", la superficie ocupada es alrededor de 600 metros cuadrados, consta con una oficina en donde estará el servidor para el sistema de monitoreo.

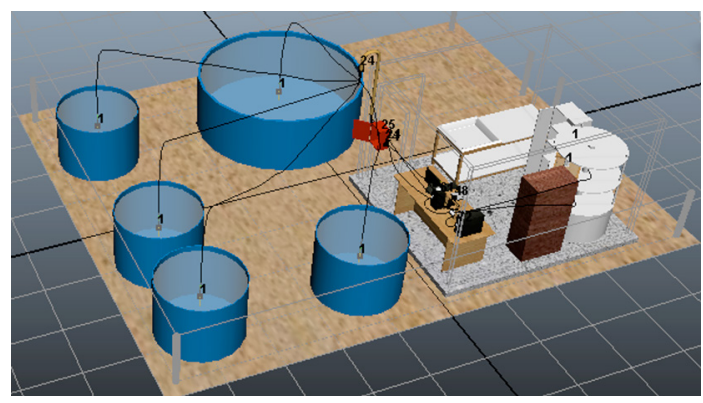

Figura 4. Laboratorio con sistema de monitoreo vista lateral. Fuente propia.

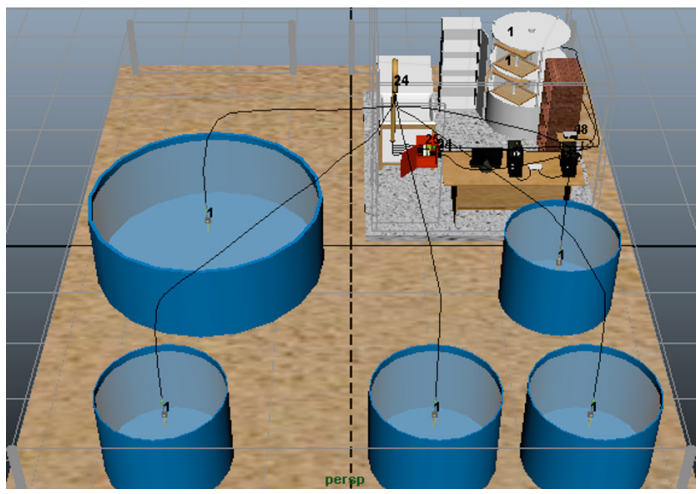

Figura 5. Laboratorio con sistema de monitoreo vista frontal. Fuente propia.

\section{Diagrama de colaboración del sistema}

Como parte del trabajo de diseño en la Figura 6 se muestra el diagrama general del sistema, se observa como las principales solicitudes que se realizan al nodo recolector. La petición manual de datos puede gatillarse por dos razones, una solicitud de datos de alertas o una solicitud de datos actuales, hechas por dos subsistemas distintos, el resultado de cada una respectivamente, es una notificación al correo de los usuarios con un mensaje sobre variables que exceden el rango permitido, y a través de una de 


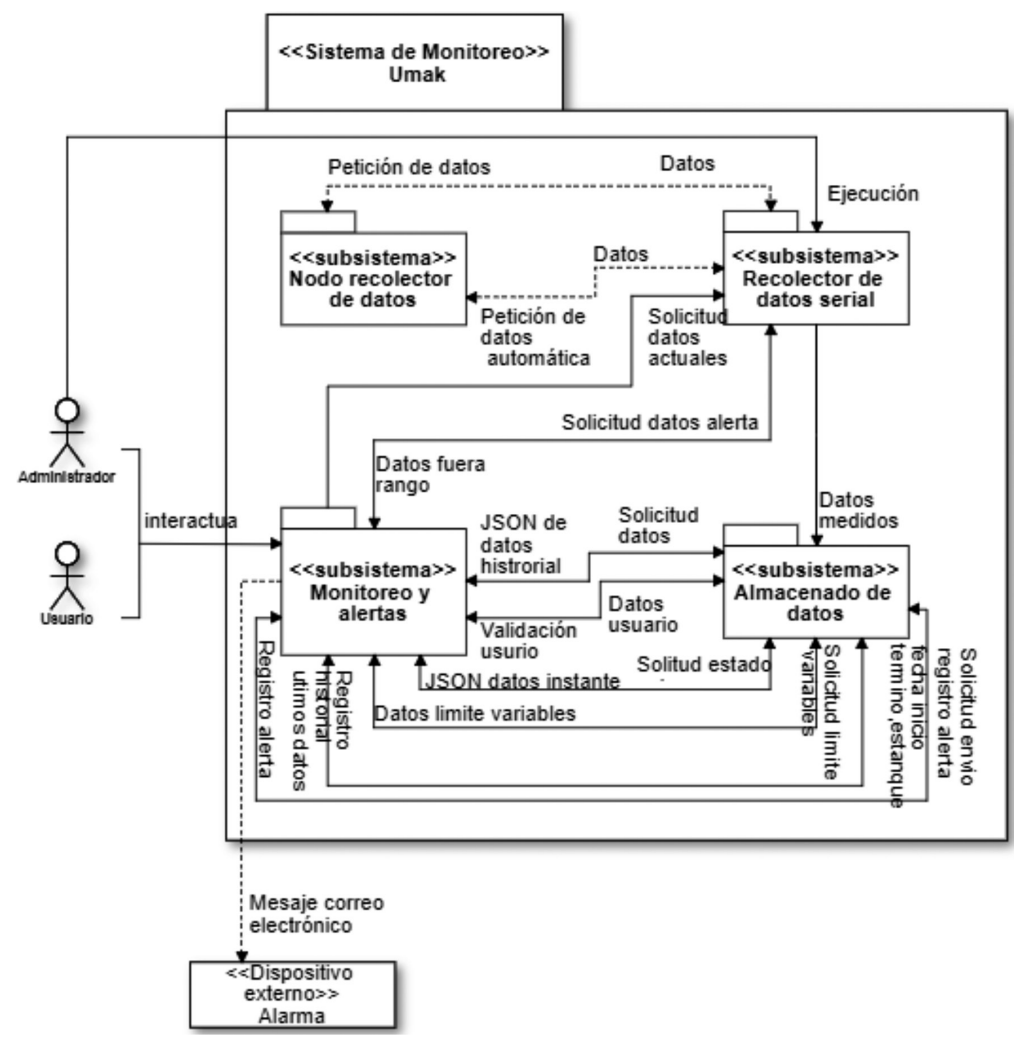

Figura 6. Diagrama de colaboración general. Fuente propia.

las vistas del sistema la posibilidad de observar el estado actual del estanque.

\section{Diseño de la red Zigbee}

La Figura 7 muestra el sistema de comunicación de los nodos de captura de datos implementados utilizando Zigbee inalámbrica, con topología estrella. El coordinador debe estar conectado a un puerto USB del servidor. Los dispositivos XBee finales se conectan al coordinador, de manera inalámbrica, para transferir los datos de lecturas captados por los sensores del nodo donde se encuentran integrados.

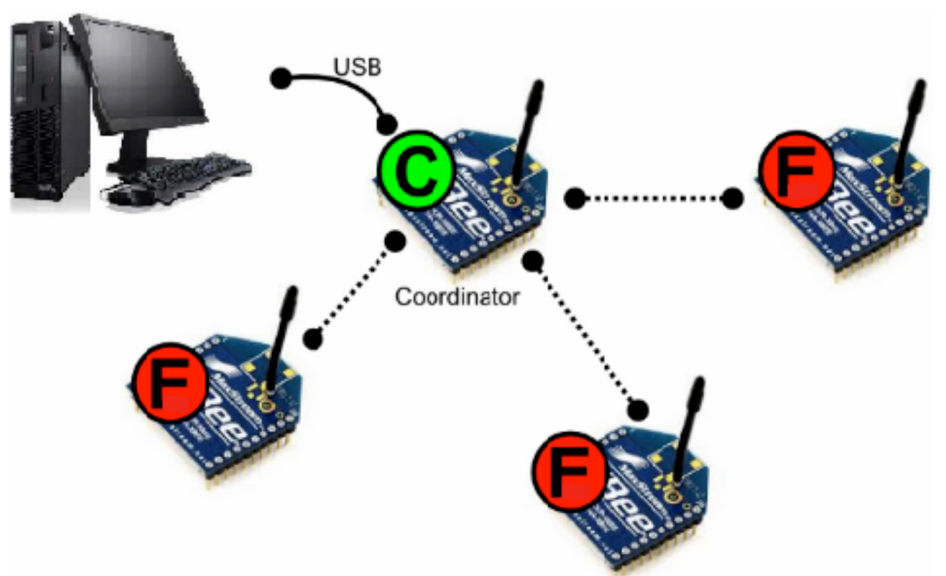

Figura 7. Red Zigbee. Fuente propia. 


\section{ANÁLISIS DE RESULTADOS}

Para comprobar el funcionamiento del monitoreo del estanque en tiempo real, se realizaron pruebas a través de un Smartphone Samsung mini 3 modelo GT-I8190L con versión Android 4.1.2. Todas las funcionalidades probadas desde un computador no tienen problemas, por esto, las pruebas que se muestran en esta sección se realizaron desde un celular.

Usando un navegador Chrome con la última versión instalada en el celular, todos sus componentes cargaron sin problemas, como se muestra en la Figura 8.

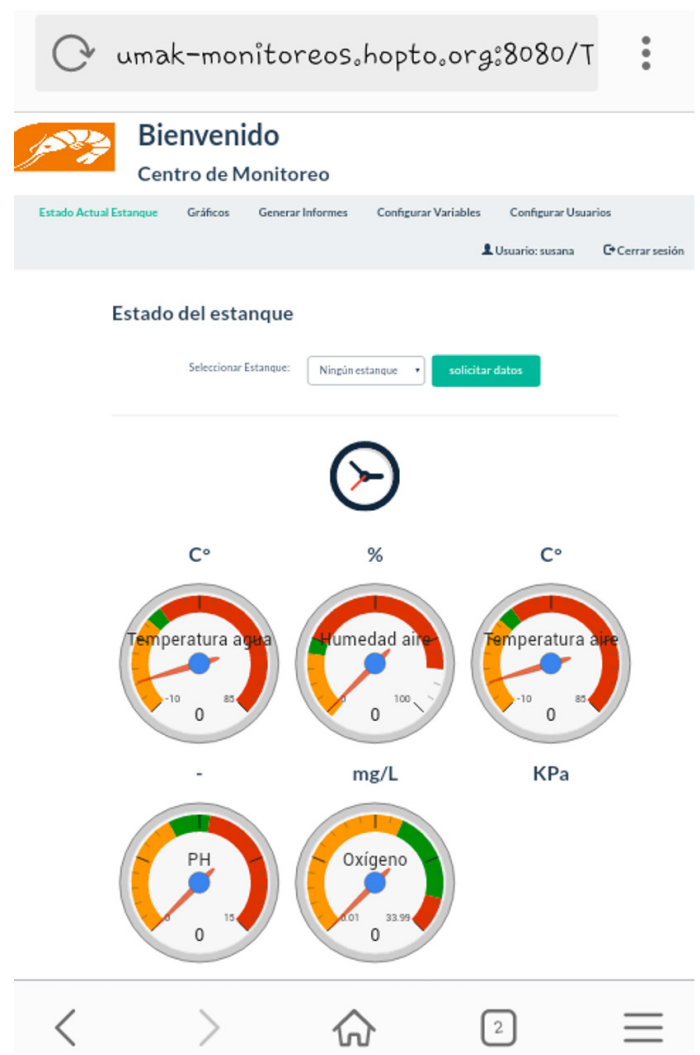

Figura 8. Estado actual del estanque vista Smartphone. Fuente propia.

El nodo recolector de datos fue puesto en el ambiente de los estanques, la ubicación en donde se encuentra se observa en la Figura 9.

\section{Prueba sensor de temperatura}

Comparando la lectura de un termómetro para el agua y la lectura del nodo, estos marcan $26^{\circ} \mathrm{C}$
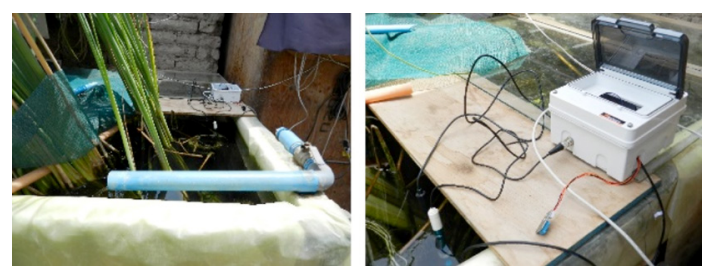

Figura 9. Nodo recolector en Estanque. Fuente propia.

en un mismo estanque, esto indica que el nodo está enviando datos correctamente con el sensor DS18B20, como muestra la Figura 10.

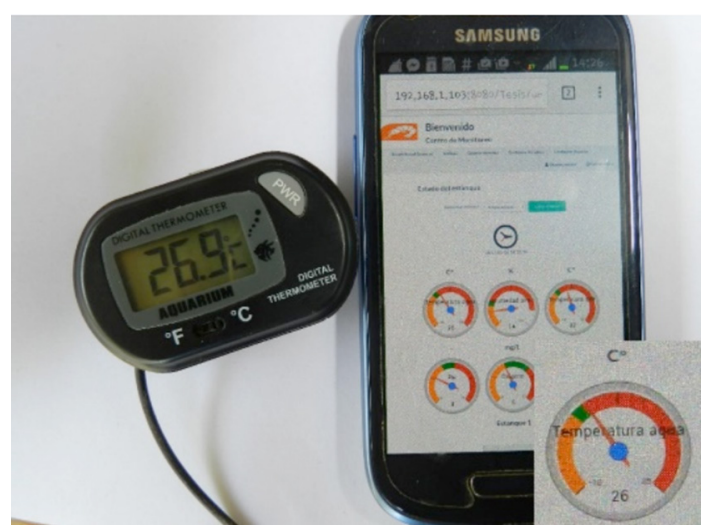

Figura 10.Comparación manual real de la temperatura. Fuente propia.

En la Tabla 1 se observa la diferencia entre las lecturas captadas por los diferentes dispositivos, durante un minuto mantuvieron sus respectivas lecturas y con una diferencia de error aceptable, debido a que el margen de error del sensor es de $\pm 1^{\circ} \mathrm{C}$, entonces 0,9 estaría dentro del rango.

Tabla 1. Diferencia entre lectura manual y sensor temperatura agua. Fuente propia.

\begin{tabular}{|c|c|c|}
\hline Lectura manual & Lectura sensor & Diferencia \\
\hline $26,9^{\circ} \mathrm{C}$ & $26^{\circ} \mathrm{C}$ & 0,9 \\
\hline
\end{tabular}

\section{Prueba sensor de oxígeno disuelto del agua}

Utilizando un medidor de oxígeno manual que se muestra en la Figura 11 se comparó con las lecturas obtenidas del sensor de oxígeno disuelto Atlas Scientific. En la Figura 12, se puede observar la 
captura de datos realizada por los dos dispositivos al mismo tiempo, ambos marcaron $5 \mathrm{mg} / \mathrm{l}$ en un mismo estanque, probando que la medición del sensor del nodo funciona correctamente.

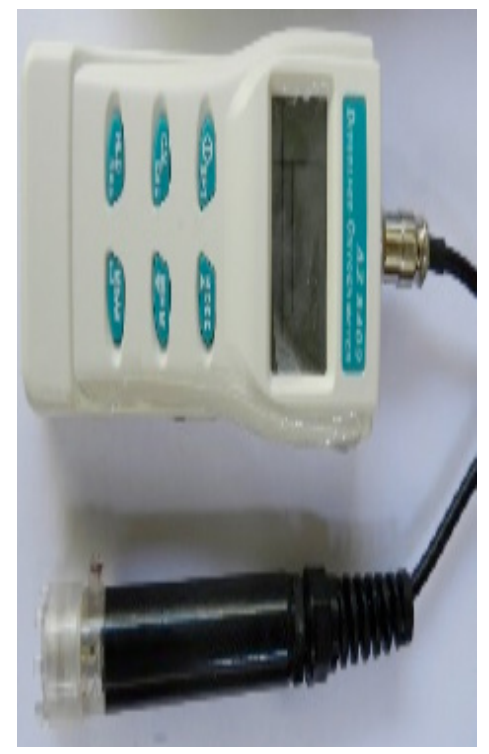

Figura 11. Medidor manual oxígeno disuelto. Fuente propia.

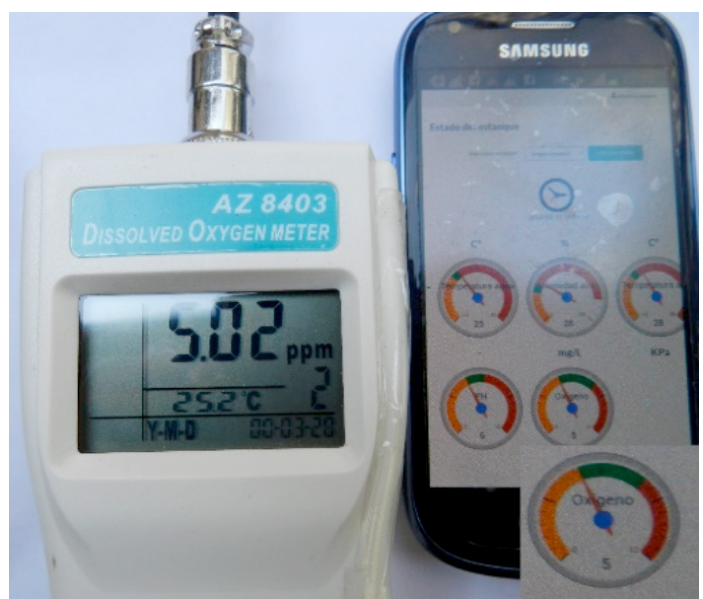

Figura 12. Comparación oxígeno disuelto en el agua. Fuente propia.

En la Tabla 2 se observa la diferencia entre los dispositivos. Durante un minuto mantuvieron sus respectivas lecturas con una diferencia de error aceptable, debido a que el margen de error del sensor es de $\pm 0.05 \mathrm{mg} / \mathrm{l}$.
Tabla 2. Diferencia entre lectura manual y sensor oxígeno disuelto. Fuente propia.

\begin{tabular}{|c|c|c|}
\hline Lectura manual & Lectura sensor & Diferencia \\
\hline $5.02 \mathrm{mg} / \mathrm{l}$ & $5 \mathrm{mg} / \mathrm{l}$ & 0.02 \\
\hline
\end{tabular}

\section{Prueba sensor de $\mathbf{p H}$}

Para comprobar la captura de datos del sensor de $\mathrm{pH}$ SEN0161 se utilizó un medidor de PH manual. Los dos dispositivos marcaron 7 en el mismo estanque como se muestra en la Figura 13, no obstante, el sensor SEN0161 ante una variación muy extrema, no funciona correctamente, capturando valores en un rango de error más amplio. Esto se debe a que el sensor es un dispositivo que no está diseñado para trabajar en un ambiente rústico, más bien, con fines académicos. Aun así, se demostró que es posible integrar un sensor de $\mathrm{pH}$ al Arduino, para hacer capturas continuas, sólo es necesario adquirir un dispositivo de mayor calidad y de mayor valor.

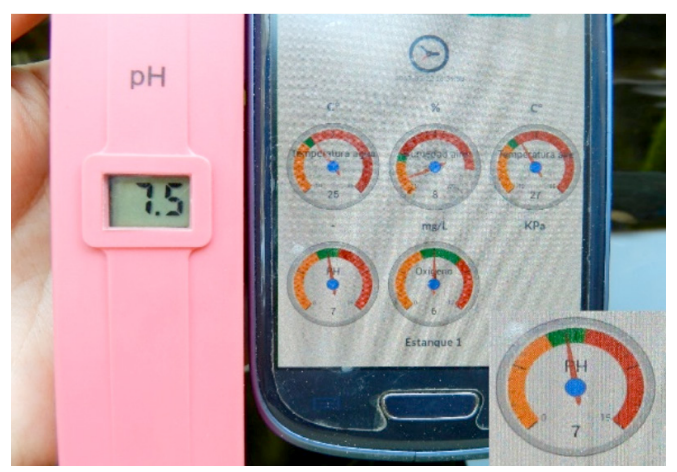

Figura 13. Comparación pH. Fuente propia.

La Tabla 3 muestra las lecturas capturadas durante un minuto, donde la diferencia entre los dos dispositivos fue muy variada, un rango de error no aceptable, ya que el margen de error del sensor es de $\pm 0.1 \mathrm{pH}$, una lectura con diferencia $3,5 \mathrm{pH}$ indica que el sensor captura datos erróneos.

Tabla 3. Diferencia entre lectura manual y sensor pH. Fuente propia.

\begin{tabular}{|c|c|c|}
\hline Lectura manual & Lectura sensor & Diferencia \\
\hline 7.5 & 7 & 0.5 \\
\hline 7.5 & 8 & 0.5 \\
\hline 7,5 & 10 & 2.5 \\
\hline 7,5 & 4 & 3,5 \\
\hline
\end{tabular}




\section{Conclusiones de las pruebas}

Las pruebas realizadas no demostraron errores con respecto a la programación del sistema, no obstante, se debe tomar en cuenta los navegadores que se utilizan, Chrome tiene el mejor funcionamiento en celulares y computadores, siempre usando las últimas versiones. El navegador Safari para celular tiene mejores resultados en la versión IPhone, no así en sistema Android o computador. Opera es otro navegador en que las funciones se ejecutan correctamente desde un computador, no obstante, en un celular con Android versión 4.1.1 no lo hace perfectamente. La mejor opción es utilizar Chrome, ya sea en un computador de escritorio o celular para acceder al sistema.

Algunos sensores mostraron errores en la captura de datos, demostrando que la precisión en la captura se ve fuertemente reflejado por la calidad del sensor, principalmente con el sensor de $\mathrm{pH}$, humedad y temperatura ambiental, siendo éstos los de menor costo en su categoría. En el caso de la medición del oxígeno disuelto, se utilizó un sensor especializado para trabajar en entornos más complejos, sensor de un costo más elevado, con resultados más precisos al capturar datos.

\section{CONCLUSIONES}

En este proyecto se desarrolló un sistema de monitoreo con acceso remoto de las condiciones del agua de los estanques y del ambiente de un criadero de camarones. El software entrega herramientas que permiten al usuario visualizar datos del ambiente a través del tiempo, con el fin de analizar el comportamiento y prevenir condiciones críticas del medio acuático en el que vive el camarón de río.

Para llevar a cabo el proyecto no sólo se utilizaron herramientas del área de la informática, sino que también se interactuó con profesionales de acuicultura y mecánicos, con el objetivo de desarrollar una aplicación real y no simulada.

Los sensores y dispositivos manuales que se utilizaron para la captura de los datos del agua fueron los más complejos de seleccionar, se debía integrar al Arduino y ser resistentes al agua. El microcontrolador Arduino, fue seleccionado por su capacidad de integrar otros dispositivos con mayor facilidad como la comunicación inalámbrica y facilidad para crear un nodo con bastante potencial de independencia del lugar de trabajo.

$\mathrm{Al}$ integrar las tecnologías descritas anteriormente se obtuvo un prototipo, que demostró ser eficaz y necesario de aplicar en lugares donde se requiere llevar un control preciso de las condiciones ambientales.

Trabajo futuro: se debe mejorar las notificaciones de alerta de los niveles del agua fuera de rango, integrando al nodo recolector de datos un Sistema Global para Comunicaciones Móviles (GSM), dándole la capacidad al nodo de conectarse a internet usando una tarjeta SIM.

Una de las principales mejoras que se pueden realizar al sistema, está en reemplazar los sensores con fines académicos, por sensores especializados para el trabajo en terreno, con el propósito de aumentar la precisión del monitoreo.

Para darle más independencia al nodo recolector de datos, la fuente de alimentación debe ser cambiada por baterías, que sea capaz de recargarse con el sol, implicando que el funcionamiento del nodo sea programado para funcionar de manera eficiente.

\section{AGRADECIMIENTOS}

Se agradece el apoyo del Proyecto de Investigación para Estudiantes de Pregrado $N^{\circ} 8724-16$ de la Universidad de Tarapacá. Además, a los Ingenieros Víctor Flores, Cristián Flores y familia quienes prestaron sus instalaciones y conocimientos en acuicultura.

\section{REFERENCIAS}

[1] FAO, Producción en acuicultura. URL: http://www.fao.org/fishery/countrysector/ naso_chile/es. Fecha de Consulta: 09 de mayo de 2018.

[2] Mundo acuícola. URL: http://mundoacuicola.cl/?/ revista/144/diversificacion-acuicola-en-chile/2. Fecha de consulta: 19 de enero de 2017.

[3] J. Sandnes, T. Ringstad, D. Wenner, P. Heyerdahl, T. K"allqvist y H. Gislerød, Real-time monitoring and automatic density control of large-scale microalgal cultures 
using near infrared (NIR) optical density sensors. Journal of Biotechnology. 2009.

[4] K. Bhuiya, K. Anish, D. Parekh y K. Laxmi, Low Cost Wireless Control And Monitoring Using PLC and SCADA, Int. Journal of Scientific and Research Publications, 2012.

[5] B.Zion, The use of computer vision technologies in aquaculture - A review, Computers and Electronics in Agriculture, 2013.

[6] J.Mathiassen, E. Misimi, M. Bondo, E. Veliyulin y S. Ostvik. Trends in application of imaging technologies to inspection of fish and fish products, Trends Food Sci. Technol, 2011.

[7] J.A. Meruane1, M.C. Morales, C.A. Galleguillos, M.A. Rivera y H. Hosokawa. Experiencias y resultados de investigaciones sobre el camarón de río del norte cryphiops caementarius (molina 1782) (decapoda: palaemonidae): historia natural y cultivo. Scielo, 2006. URL: http://www.scielo. c1/scielo.php?script=sci_arttext\&pid $=$ S0717-65382006000200015. Fecha de consulta: 19 de enero de 2017.

[8] M.C. Morales y J. Meruane. Indicadores de condición larvaria aplicados al camarón de río del norte Cryphiops caementarius (Molina, 1782), en condiciones de cultivo controlado. Scielo, 2012. URL: http://www.scielo.cl/ scielo.php?script=sci_arttext\&pid=S0718560X2012000300020. Fecha de consulta: 19 de enero de 2017.

[9] C. Yávar y E. Dupré. Desarrollo embrionario del camarón de río Cryphiops caementarius (Decapoda: Palaemonidae) en condiciones de laboratorio. OTS, 2007. URL: http://www. ots.ac.cr/rbt/attachments/suppls/sup55-1\%20 crustacea/03-YAVAR.pdf. Fecha de consulta: 20 de enero de 2017.

[10] Processing. URL: https://processing.org/. Fecha de Consulta: 20 de febrero de 2017. 